\title{
Introducing a New Activity-Based Balance Index Using Accelerometer Data and Evaluating it as a Predictor of Skill Level among Elite Junior Soccer Players ${ }^{+}$
}

\author{
Shahram Lenjannejadian ${ }^{1, *}$, Jalil Reisi ${ }^{2}$ and Mehdi Salimi ${ }^{3}$ \\ 1 Department of Sport Injuries, Faculty of sport sciences, University of Isfahan, Isfahan, Iran \\ 2 Department of Exercise Physiology, Faculty of sport sciences, University of Isfahan, Isfahan, Iran; \\ jalil_reisi@yahoo.com \\ 3 Department of Sport Management, Faculty of sport sciences, University of Isfahan, Isfahan, Iran; \\ m_salimi_3@yahoo.com \\ * Correspondence: shahram_lenjani@yahoo.com; Tel.: +98-913-327-6826 \\ + Presented at the 13th conference of the International Sports Engineering Association, Online, 22-26 June \\ 2020.
}

\begin{abstract}
The aim of this study was to introduce a new activity-based balance index by using accelerometer data. Twenty-seven junior soccer players from the Iranian premier league were selected. Four functional tests, consisting of one leg stance, dynamic $\mathrm{Y}$ balance, running and dribbling tests, were conducted to assess the players' balance, activity and skill. During these four tests, besides their relative scores, the acceleration of their body center was also recorded. Activitybased balance index (ABI) was calculated using these acceleration data. The results showed positive correlations between $\mathrm{ABI}$ and both static and dynamic balance scores. Additionally, negative correlations were found between ABI and dribbling scores, which demonstrate the agility required for this skill. It seems that this new index achieves the evaluation of both the balance and the skill level of soccer players. Perhaps this is a new way of talent identification and also a re-development of balance tests from traditional to modern.
\end{abstract}

Keywords: activity-based balance index; soccer; accelerometer; performance; skill assessment

\section{Introduction}

The balance of an object is defined by the summation of all forces and torques applied to the object become zero [1]. In standing position, this definition can be considered as lesser displacement of the center of pressure around the balance point. Similarly, in walking, the oscillations of the center of pressure around the foot axis can be referenced as an indicator of balance. Researchers defined the balance as the ability to maintain center of gravity in the center of pressure zone between two legs [2]. Stability is also defined as the resistance to balance changes [3]. It seems that measuring this oscillation during an activity could result in a better functional assessment of balance.

There are many methods of measuring and the evaluation of the balance and stability used by researchers in laboratory and field conditions. Several tests, such as berg balance, functional reach, up and go, Y and star dynamic balance tests are often used [4,5]. Recently, advanced laboratory equipment has also been used to assess the balance quantitatively and precisely. One of these advanced equipment types is force plate. The high cost of force plates and their complicated mounting requirements in specific spaces limit their applicability in many situations. Fortunately, there are other state-of-the-art techniques, and devices could be used in different conditions. One of these is the accelerometer. By using the accelerometer data accurately, it is possible to assess balance not only in the laboratory, but also in many sport skills. Moreover, it has a reasonable price; it is a non-invasive device, and it is easy to use. Researchers compared the results of two different posture stability measurement methods, the force plate and the accelerometer. They reported that there was no difference between these two methods and both devices showed similar results in this regard [3]. 
Soccer players do various activities such as running forward and backwards with different speeds, head butting, turning around, tackling and throwing. Therefore, there is a lot of situations which can cause different injuries among players. This is especially true for non-professional athletes, which increases the likelihood of injuries such as ankle sprain and rupture of the anterior cruciate ligament and meniscus [6]. One of the important reasons for such injuries is the lack of postural control among soccer players [7]. Therefore, evaluation of the balance of a player while performing a skill will be a great help to coaches and athletes, to improve their performance, as well as predicting and preventing sports injuries. This study introduces a new index for evaluating the balance during activity using accelerometer. Additionally, it investigates how well this index can assess the balance in static and dynamic conditions, and during the performance of soccer skills.

\section{Materials and Methods}

To calculate and evaluate a new index for balance assessment in different conditions, including static standing, during activity, and during skill performance, four different tests were administered on a total of 27 junior soccer players. The participants were the players of U-14 and U-15 teams of Sepahan Football Club, who participated in the Iranian Premier League. The mean of their age is 14.5 years, height is $172.2 \mathrm{~cm}$, mass is $58.0 \mathrm{~kg}$ and their average professional experience is 4.5 years.

The tests included static balance performed on Footscan, $\mathrm{Y}$ dynamic balance, running on Footscan, as well as dribbling. During all these four tests, the acceleration of the center of the body was recorded using an accelerometer, of which the sampling rate was set to a frequency of $1600 \mathrm{~Hz}$. This triaxial accelerometer has a range of $\pm 16 \mathrm{~g}$ and 13-bit resolution. It was mounted on the lumbar region via a waist belt. In two static balance and running tests, the center of pressure (COP) positions in mediolateral and anteroposterior directions were measured. Besides, the test score of the $Y$ dynamic balance, as the normalized average reach in three directions of anterior, posterior medial and posterior lateral was computed. Finally, the time of completing the dribbling test was measured.

\subsection{Balance Evaluation in Four Selected Tests}

In the static balance test, the player was required to stand on his toes for $10 \mathrm{~s}$ on Footscan, then his COP positions were measured and recorded. In Y dynamic balance test, the main goal was to maintain single-leg balance. The players were asked to reach as far as possible with the contralateral leg in three different directions, including anterior, posteromedial and posterolateral. This was performed on each leg. The maximum distances of players' reach were measured and normalized by the height. In the running test, the participant was required to run $10 \mathrm{~m}$, while he was asked to put his dominant foot on Footscan, which was approximately laid at the middle of the $10 \mathrm{~m}$ route. The $\mathrm{COP}$ of his landing foot in mediolateral and anteroposterior directions were measured. Finally, in the dribbling skill test, the player was standing at $9 \mathrm{~m}$ from the first cone. By the start command, he was required to run with the ball through the cones in a zigzag route. Then, after finishing the route, he was required to return back to the starting point. The time of completing the test was measured. A total of two seconds was added to the measured time of the player, in case of any fouls he committed during the test.

\subsection{Calculating the Balance Index}

As stated before, the oscillation of COP is an accepted concept for defining the balance, and because this oscillation could be intuitively and objectively understood, we defined a new activitybased balance index. Oscillations of movement-related parameters over their optimal values were quantitatively calculated. The movement-related parameters could be consisted of each object's position, or its first and second derivations, known as velocity and acceleration. For computing the activity-based balance index, these movement-related signals must be measured or calculated first. Two terms $-A(t)$, which stands for the activity signal, and $I(t)$, which stands for the Index signalwere considered. The Index and activity signals were extracted by the low pass filter of the main measured signal, with respectively low and high cut-off frequencies. Choosing the frequency values 
depend on the activity or skill type. These two low and high frequencies were called activity and index frequencies in this study and they were shown as FA and FI respectively. Based on this definition, the activity-based balance index could be quantified by developing the following equation:

$$
A B I=\frac{1}{T} \int_{t_{i}}^{t_{f}} \frac{1}{I(t)} \sqrt{[I(t)-A(t)]^{2}} d t
$$

The difference of $I(t)$ and $A(t)$ in each time interval was first normalized and considered as a subindex. The average value of all sub-indices during the complete time of the activity was defined as activity-based balance index, stated as a percentage of the index signal. Therefore, the zero value of the index implies that these two signals have no difference. In other words, the activity or performance is interpreted as desired or perfect. However, the index value of activity could vary from zero to any large number.

\subsection{Data Analysis}

As stated before, the assessment signal could be any of the movement-related variables, like acceleration, velocity and position. In this study, all of these variables were considered, but only acceleration and positions are reported. The acceleration data were recorded by a sensor (Figure 1) and integrated mathematically to calculated velocity and position.
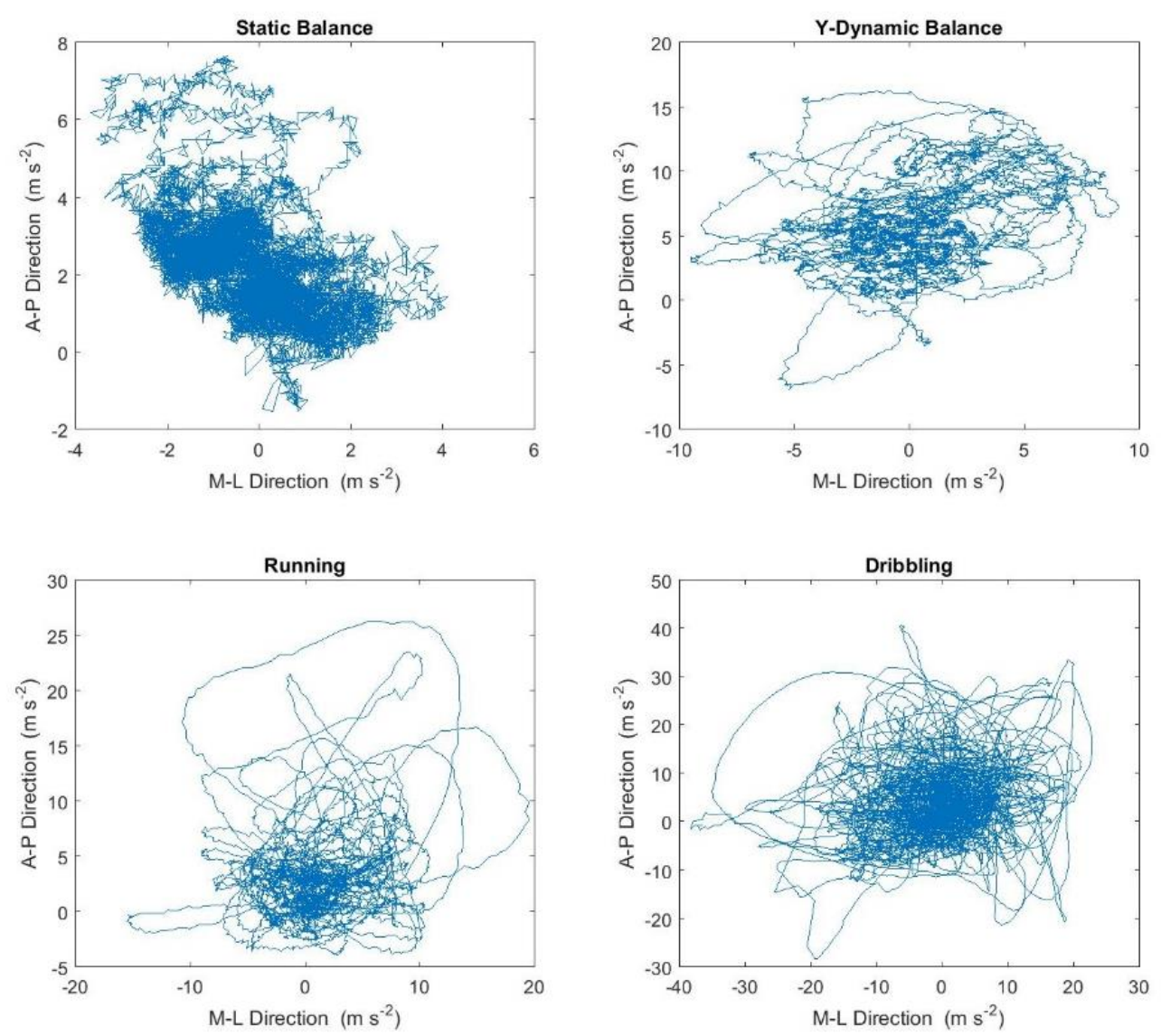

Figure 1. Acceleration of body center in Mediolateral (M-L) and Anteroposterior (A-P) directions during four selected skills.

The raw data of these signals were processed with many combinations of activity and index frequencies to calculate the corresponding activity-based index according to Equation (1). The effectiveness of these calculated indices in different four selected tests were assessed by computing the correlation coefficient of these indices with COP, score and time of each four tests respectively. Several combinations of activity and index frequencies were considered to achieve a better correlation coefficient for all four experimental tests. 


\section{Results}

The results of four selected experimental tests are shown in Table 1 . The average and standard deviation of dribbling time, COP movement in antro-posterior and medio-lateral directions during both static balance and running tests, and the score of the $\mathrm{Y}$ balance test, are shown in this table.

Table 1. Results of four activity and skill tests.

\begin{tabular}{lcc}
\hline \multicolumn{1}{c}{ Parameters } & $\begin{array}{c}\text { Measurement } \\
\text { Unit }\end{array}$ & $\begin{array}{c}\text { Mean } \\
\text { (SD) }\end{array}$ \\
\hline $\begin{array}{l}\text { Dribbling time } \\
\text { Static Balance } \\
\quad \text { Center of pressure (COP) Movement in }\end{array}$ & Second & $12.7(1.0)$ \\
anteroposterior (AP) Direction & Millimeter & 117.0 \\
$\quad$ COP Movement in medio-lateral (ML) & Millimeter & $78.2(75.4)$ \\
Direction & & 1041.8 \\
$\quad$ Total COP Movement & Millimeter & $(501.2)$ \\
& & \\
Y Balance & \% of Height & $51.9(7.4)$ \\
$\quad$ Anterior Direction Score & \% of Height & $63.2(5.5)$ \\
$\quad$ Posterior Lateral Direction Score & \% of Height & $61.3(6.2)$ \\
$\quad$ Posterior Medial Direction Score & \% of Height & $58.8(5.5)$ \\
$\quad$ Overall Score & Millimeter & $31.2(9.4)$ \\
Running & Millimeter & 224.3 \\
$\quad$ ML COP Movement for Right Support Foot & $(52.6)$ \\
AP COP Movement for Right Support Foot & Millimeter & $36.7(11.3)$ \\
$\quad$ ML COP Movement for Left Support Foot & Millimeter & 233.2 \\
AP COP Movement for Left Support Foot & $(56.8)$ \\
\hline
\end{tabular}

During each test, at the same time, the acceleration of body center was also measured and the indices were calculated. To demonstrate the effect of choosing different values of FI and FA on numerical value of calculated movement-related indices, the values of indices for different frequencies are shown in Table 2. In the first three columns, the effect of increasing index frequency and, in the second three columns, the effect of increasing the activity frequency, could be seen.

Table 2. Mean of acceleration, and position indices during static balance test, according to different combinations of activity and index frequencies.

\begin{tabular}{lcccccc}
\hline Activity Frequency $\mathbf{( H z )}$ & $\mathbf{5}$ & $\mathbf{5}$ & $\mathbf{5}$ & $\mathbf{2}$ & $\mathbf{7}$ & $\mathbf{1 0}$ \\
\hline Index Frequency (Hz) & $\mathbf{1 0 0}$ & $\mathbf{5 0 0}$ & $\mathbf{1 0 0 0}$ & $\mathbf{5 0 0}$ & $\mathbf{5 0 0}$ & $\mathbf{5 0 0}$ \\
\hline $\begin{array}{l}\text { Acceleration Index } \\
\quad\end{array}$ & & & & & & \\
$\quad$ AP Direction & 154.9 & 158.2 & 158.6 & 214.4 & 124.2 & 84.6 \\
$\quad$ ML Direction & 168.6 & 172.3 & 172.7 & 219.1 & 150.6 & 118.3 \\
$\quad \begin{array}{l}\text { Position Index } \\
\quad \text { AP Direction }\end{array}$ & 0.40 & 0.41 & 0.41 & 1.4 & 0.23 & 0.11 \\
$\quad$ ML Direction & 0.38 & 0.39 & 0.39 & 1.3 & 0.22 & 0.12 \\
\hline
\end{tabular}

AP: Anteroposterior; ML: Medio-lateral.

As a key test to evaluate the efficacy of introduced indices for balance assessments, the correlation coefficient of COP movements during static balance test with indices calculated from acceleration and position signals were computed. The significant correlation of COP and each calculated index showed that using the accelerometer led to the same results as COP variations measured by Footscan or Forceplate devices. These correlation coefficients are shown in Table 3. 
Table 3. The correlation coefficients and their significance between indices and COP movements during static balance test, using different activity and index frequencies.

\begin{tabular}{|c|c|c|c|c|c|c|c|c|c|}
\hline \multirow[b]{3}{*}{$F A \mathcal{E} F I$} & \multicolumn{9}{|c|}{ COP Movement Range in Different Directions } \\
\hline & AP & ML & Total & AP & ML & Total & AP & ML & Total \\
\hline & \multicolumn{3}{|c|}{$F A, F I=(2,500)$} & \multicolumn{3}{|c|}{$F A, F I=(5,500)$} & \multicolumn{3}{|c|}{$F A, F I=(10,500)$} \\
\hline \multicolumn{10}{|l|}{ Acc. Index } \\
\hline AP Direction & $0.547^{* *}$ & $0.655^{* *}$ & $0.556^{* *}$ & $0.507^{* *}$ & $0.616^{* *}$ & $0.536^{* *}$ & $0.591 * *$ & $0.450 *$ & $0.648^{* *}$ \\
\hline ML Direction & 0.478 * & $0.609 * *$ & $0.537^{* *}$ & $0.533 * *$ & $0.531 * *$ & $0.670 * *$ & 0.319 & $0.447^{*}$ & $0.491^{* *}$ \\
\hline \multicolumn{10}{|l|}{ Position Index } \\
\hline AP Direction & $0.386^{*}$ & $0.658^{* *}$ & $0.459 *$ & 0.470 * & $0.720^{* *}$ & $0.547^{* *}$ & $0.532 * *$ & $0.702 * *$ & $0.583^{* *}$ \\
\hline ML Direction & 0.463 * & $0.500 * *$ & $0.504^{* *}$ & $0.525^{* *}$ & 0.379 & $0.566^{* *}$ & $0.484^{*}$ & 0.401 * & $0.579^{* *}$ \\
\hline
\end{tabular}

The main purpose of this research was to introduce an activity-based index which is capable of showing both the balance of the body during activity and also its possible relationship with performance. Therefore, it is essential to calculate the correlation coefficient of these indices with each activity test or skill. Table 3 is an example in this regard. Since there are many different choices for movement-related indices and their corresponding frequencies, only some selective results are shown in Table 4. Significant correlation coefficients showed the appropriateness of the selected index and its frequency combination.

Table 4. The values of correlation coefficients and their significance between selected indices and the results of their corresponding activity tests and skills.

\begin{tabular}{lc}
\hline \multicolumn{1}{c}{ Selected Indices } & $\begin{array}{c}\text { Correlation } \\
\text { Coefficient }\end{array}$ \\
\hline $\begin{array}{l}\text { Y Balance } \\
\text { Activity-based balance index (ABI) and Y Balance Score in AP Direction at } \\
\text { frequencies FA, FI }=(1,500)\end{array}$ & $0.601^{* *}$ \\
ABI and Total Score of Y Balance at frequencies FA, FI $=(1,500)$ & $0.473^{*}$ \\
Running & \\
ABI and ML COP Movement at frequencies FA, FI $=(2,500)$ & $0.513^{* *}$ \\
ABI and ML COP Movement at frequencies FA, FI $=(1,500)$ & $0.458^{*}$ \\
Dribbling & \\
ABI and Dribbling time at frequencies FA, FI $=(1,200)$ & $-0.453^{*}$ \\
ABI and Dribbling at frequencies FA, FI $=(1,500)$ & $-0.410^{*}$ \\
\hline
\end{tabular}

$$
{ }^{*}: p \text { value }<0.05 ; * *: p \text { value }<0.01
$$

\section{Discussion}

Balance is a completely unique phenomenon according to each specific sport performance or activity. For example, taekwondo or karate require specific balance abilities, which differ from those of wrestling, gymnastics, or group sports such as handball, volleyball, and soccer. Due to the diversity of sports, traditional static and dynamic balance tests are not appropriate for all athletes. Therefore, the activity-base balance definition seems more logical.

In general, the results showed a positive and significant correlation of acceleration and position index with COP excursion during static balance test at different movement frequencies (Table 3). In addition, the results of the correlation coefficient of activity-based balance index (ABI) with $Y$ dynamic balance and running scores showed a positive and significant correlation (Table 4). These results indicate that, in those activities that intuitively the balance ability directly affect the scores, there is also a direct relation between test score and the introduced index. It means that the ABI satisfies this intuitive sense.

It was interesting to note that there was a negative and significant correlation between the score of the balance index and the dribbling time. In other words, the results indicate that as the dribbling 
time is shorter, i.e., the player's performance is better, the score is higher. Therefore, as the fluctuations in position, acceleration and speed become higher, the performance becomes better.

\section{Conclusions}

It seems that the traditional view of balance can be improved by using modern, low-cost technologies and also by introducing new and appropriate quantitative indices. This could be helpful to many sport disciplines and any particular skills. Perhaps this is a new way of talent identification and also a guide to re-develop exercises from traditional into modern.

Author Contributions: All Authors contributed in research design, experimental procedure for data collection, processing the results and writing the final report.

Funding: This research received no external funding.

Acknowledgments: It is necessary to thank the officials, coaches and players of the soccer junior team of the Sepahan FC Club who have participated in this study.

Conflicts of Interest: There are no conflicts of interest to disclose.

\section{References}

1. Meriam, J.L.; Kraige, L.G. Engineering Mechanics: Dynamics; John Wiley \& Sons: Hoboken, NJ, USA, 2012; Volume 2.

2. LeVeau, B.F. Biomechanics of Human Motion: Basics and Beyond for the Health Professions; Slack Incorporated: Thorofare, NJ, USA, 2010.

3. Seimetz, C.; Tan, D.; Katayama, R.; Lockhart, T. A comparison between methods of measuring postrual stability: force plates versus accelerometers. Biomed. Sci. Instrum. 2012, 48, 386.

4. Matsuda, S.; Demura, S.; Uchiyama, M. Centre of pressure sway characteristics during static one-legged stance of athletes from different sports. J. Sports Sci. 2008, 26, 775-779.

5. Plysky, P.J.; Gorman, P.P.; Butler, R J.; Kiesel, K.B.; Underwood, F.B.; Elkins, B. The reliability of an instrumented device for measuring components of the star excursion balance test. N. Am. J. Sport. Phys. Ther. NAJSPT 2009, 4, 92.

6. Murphy, D.F.; Connolly, D.A.J.; Beynnon, B.D. Risk factors for lower extremity injury: a review of the literature. Br. J. Sports Med. 2003, 37, 13-29.

7. Söderman, K.; Alfredson, H.; Pietilä, T.; Werner, S. Risk factors for leg injuries in female soccer players: A prospective investigation during one out-door season. Knee Surgery Sport. Traumatol. Arthrosc. 2001, 9, 313321. 